\title{
LATE FRACTURE OF THE ACETABULUM AFTER TOTAL HIP REPLACEMENT
}

\author{
A. J. Miller, London, England \\ From the Royal Portsmouth Hospital, Portsmouth, Hampshire
}

This paper reports nine cases of fracture of the ischio-pubic region of the acetabulum occurring between fourteen and forty-eight months after total hip replacement. In only one patient was there a possibility that injury played a part.

CASE REPORTS

Case 1-An overweight woman of sixty-three with long-standing osteoarthritis of both hips was subjected to right Ring arthroplasty in May 1968: cement was used to fix the lower component. She progressed well for twelve months, but then developed right foot drop which was controlled by a toe-raising splint. Two years after operation she twisted the right leg while getting up from a settee

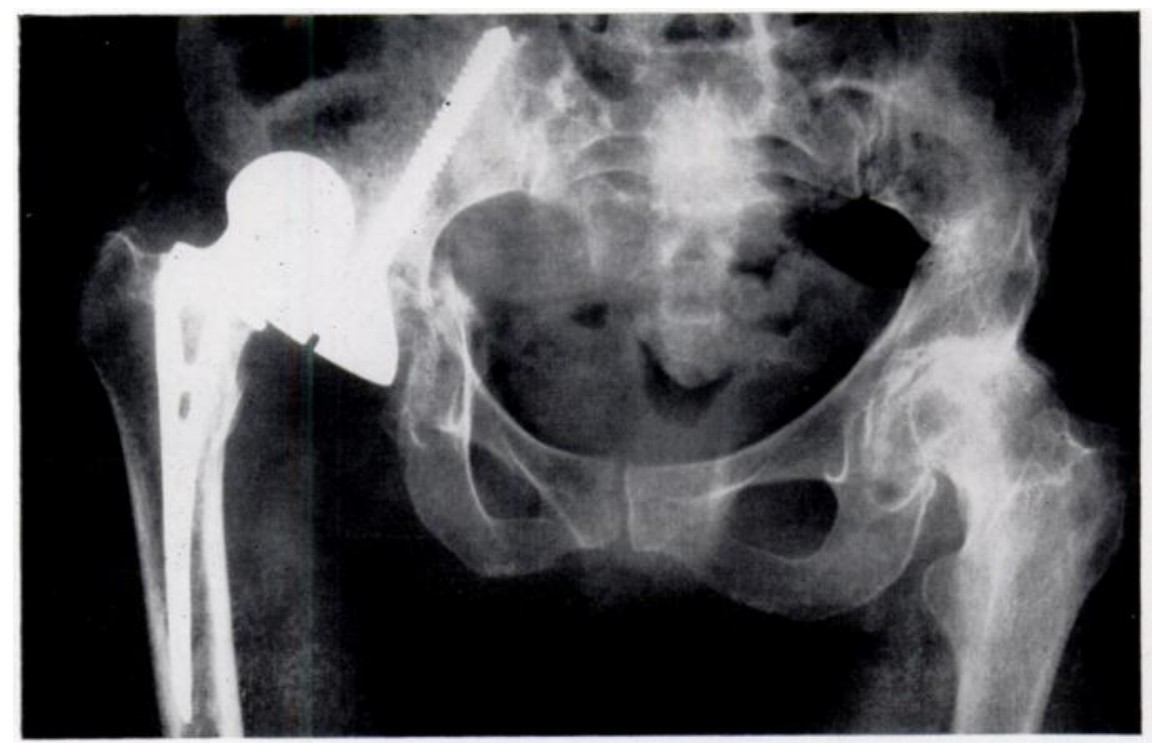

Fig. 1

Case 1-A radiograph showing the fracture of the acetabulum and dislocation of the prosthesis. An innominate osteotomy had been done previously on the left hip for arthritis.

and suffered pain in the hip. Radiographs showed a fracture through the ischio-pubic region with dislocation of the prosthesis (Fig. 1). The dislocation was reduced by open operation but recurred eight weeks later when the plaster spica was removed. In September 1970 the prosthesis was removed and the patient was left with a pseudarthrosis. The wound became infected, and a discharging sinus has persisted in spite of several operations including sequestrectomies.

Case 2-A woman aged sixty, of normal build, was treated for long-standing osteoarthritis of the left hip by Ring arthroplasty in October 1968. Progress was good for over a year until she developed a swollen left leg which was thought to be due to thrombophlebitis. Phenylbutazone was prescribed and the symptoms subsided, but she later developed foot drop. In December 1970, while sitting, she bent forwards and felt a click in the left hip. Thereafter she was unable to take any weight on the leg. Radiographs showed a fracture through the ischio-pubic region with dislocation of the prosthesis. 
Closed reduction was carried out, and the limb was supported with skin traction for six weeks. Dislocation recurred when the traction was removed and in February 1971 the prosthesis was removed. There was some wound infection but this settled down with antibiotics, and she is now walking with a frame and below-knee caliper (Fig. 2).

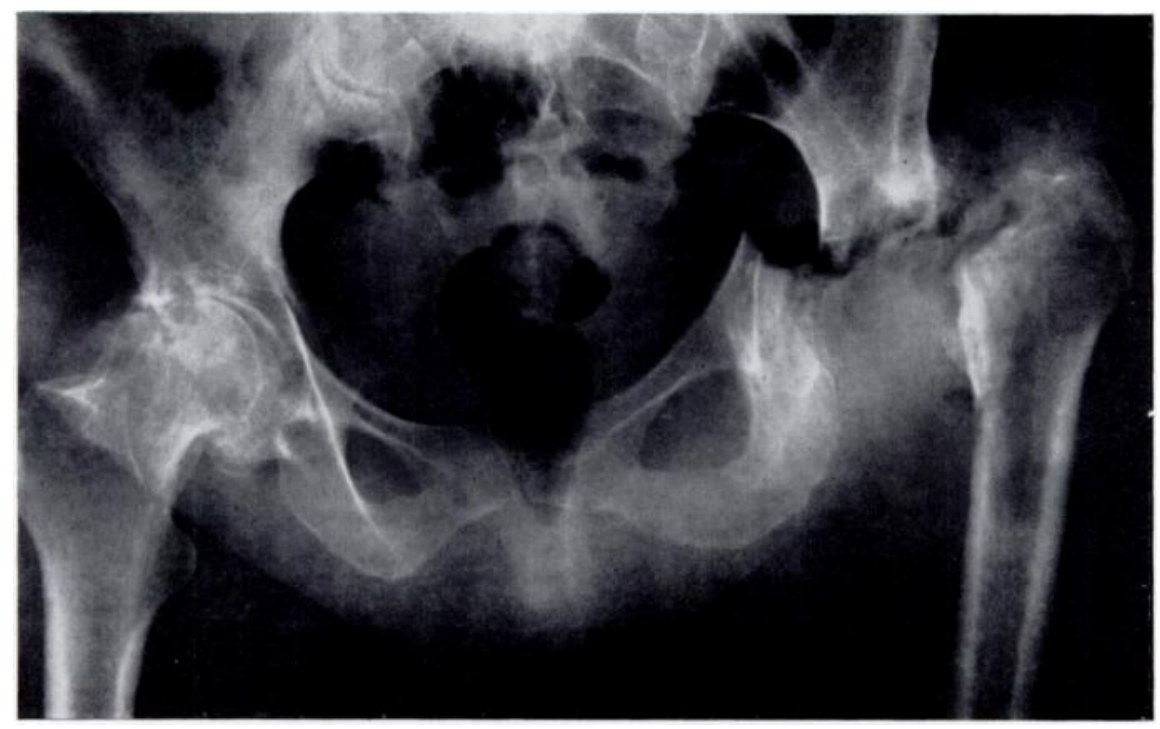

Fig. 2

Case 2-A radiograph three months after the fracture of the acetabulum which shows no evidence of union or callus formation.

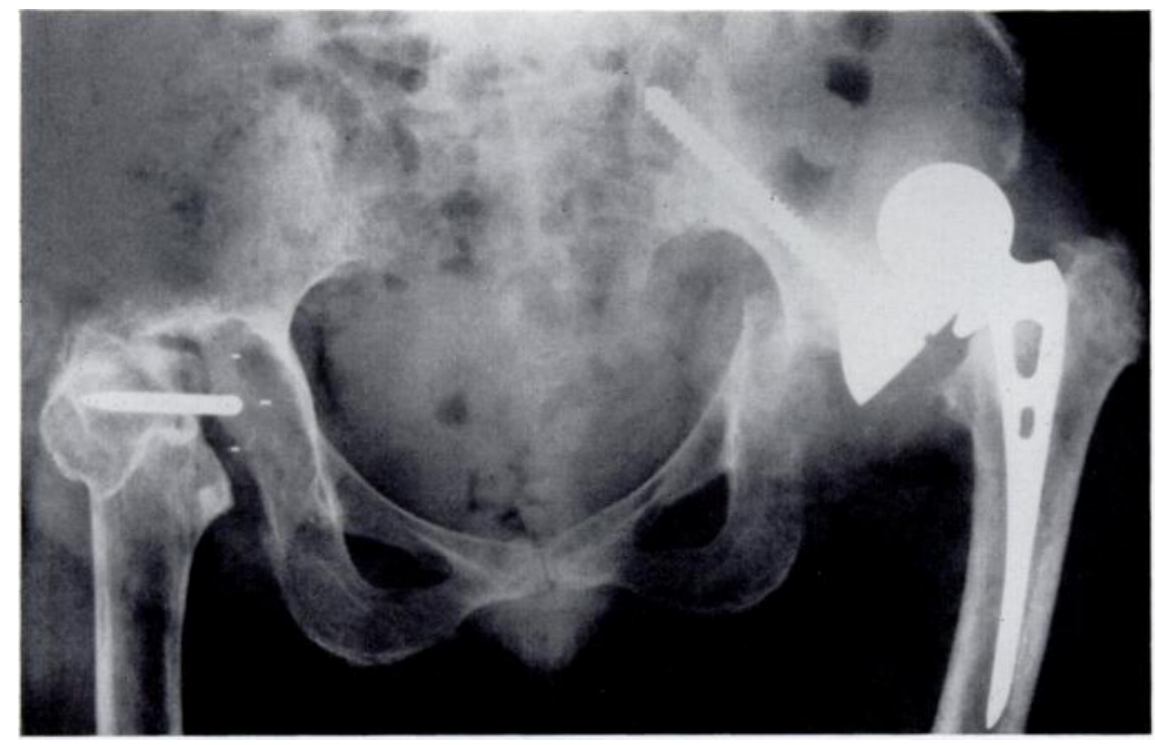

FIG. 3

Case 3-The acetabular component of the Ring prosthesis was not well countersunk and was directed too horizontally.

Case 3-An obese woman of seventy-eight with a long history of rheumatoid arthritis had in October 1968 a left Ring arthroplasty. She did well until March 1971 when, bending forwards, she felt something crunch in her left hip and her leg gave way. Radiographs showed a fracture through the 
ischio-pubic region with dislocation of the prosthesis (Fig. 3). A closed reduction was carried out but the prosthesis became dislocated again and was therefore removed. Since operation she had done very well and has now got a painless hip with good movement, and walks with two sticks.

Case 4-A woman of eighty-seven with osteoarthritis of both hips had had in 1966 a left, and in 1968 a right Ring arthroplasty. Four months after the second operation she developed an infection of the right hip. This was treated by large doses of antibiotics and drained on several occasions. The infection persisted and in February 1971 the prosthesis was removed. Radiographs showed a fracture through the ischio-pubic region (Fig. 4).

Case 5-An overweight woman aged sixty-eight was originally treated for osteoarthritis of the left hip by a Thompson prosthesis, but her pain recurred after a fall and the stem of the prosthesis was thought to be loose. In February 1967 a left McKee arthroplasty was performed. Her progress was satisfactory until August 1971 when she presented with an infection in the hip. Radiographs showed rarefaction of the ischio-pubic region of the acetabulum (Fig. 5). Despite antibiotics the infection

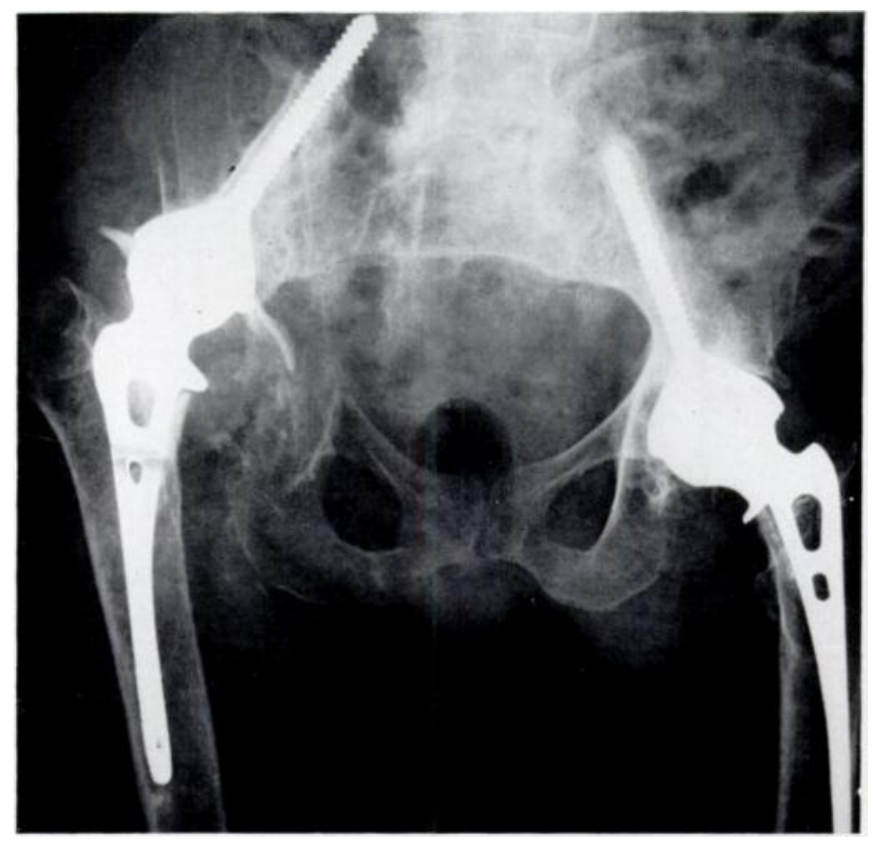

FiG. 4

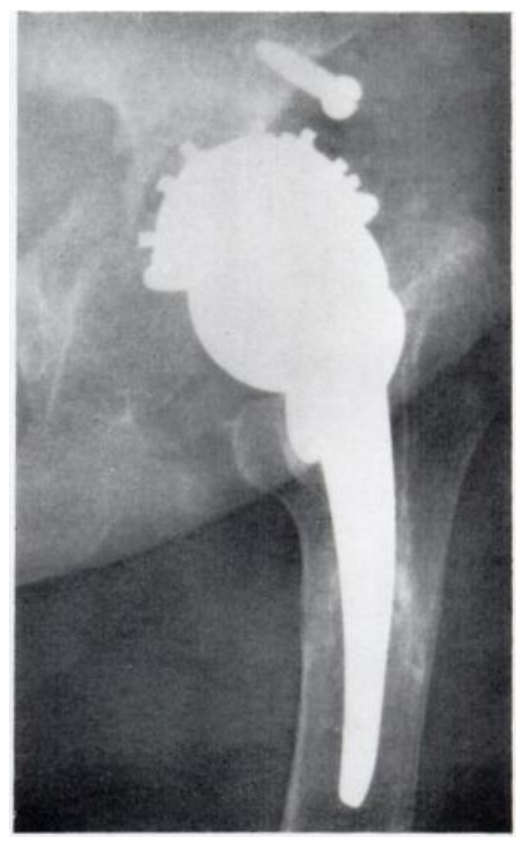

FIG. 5

Figure 4-Case 4. On the right side the acetabular component has moved proximally and the surrounding bone is disorganised. Figure 5-Case 5. A fracture has occurred after a McKee replacement.

did not settle down, a fluctuant area developed and in August 1971 the prosthesis was removed. At operation a fracture was noted in the inner wall of the acetabulum. The infection later settled leaving a small sinus.

Case 6-A woman of average weight, aged fifty-two, with osteoarthritis of her left hip was treated by left McKee arthroplasty in May 1969. Her course after operation was entirely satisfactory until January 1970 when she presented with a deep vein thrombosis in her left leg confirmed by venography (Fig. 6). This settled with bed rest and anticoagulants. She continued improving, but after a fall developed a temperature, and a few weeks later a fluctuant abscess in the operation scar. Radiographs showed a fracture through the ischio-pubic region of the acetabulum (Fig. 7). She was treated with antibiotics but failed to respond, and in April 1971 the prosthesis was removed. Difficulty was encountered in removing the cement from the femur. A persistent sinus developed and therefore re-exploration was carried out and the remaining cement removed. The hip became stable and painless, but a profuse sero-sanguinous fluid continued to discharge.

Case 7-A seventy-two-year-old woman with severe osteoarthritis of the right hip was treated in April 1969 by McKee arthroplasty. She progressed well until March 1970 when pain recurred and 


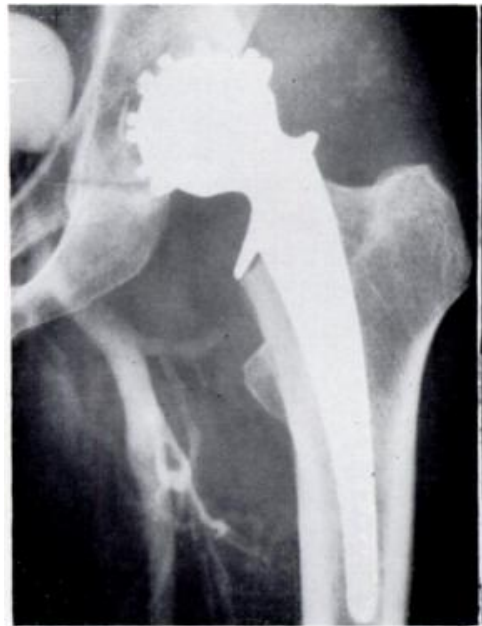

FIG. 6

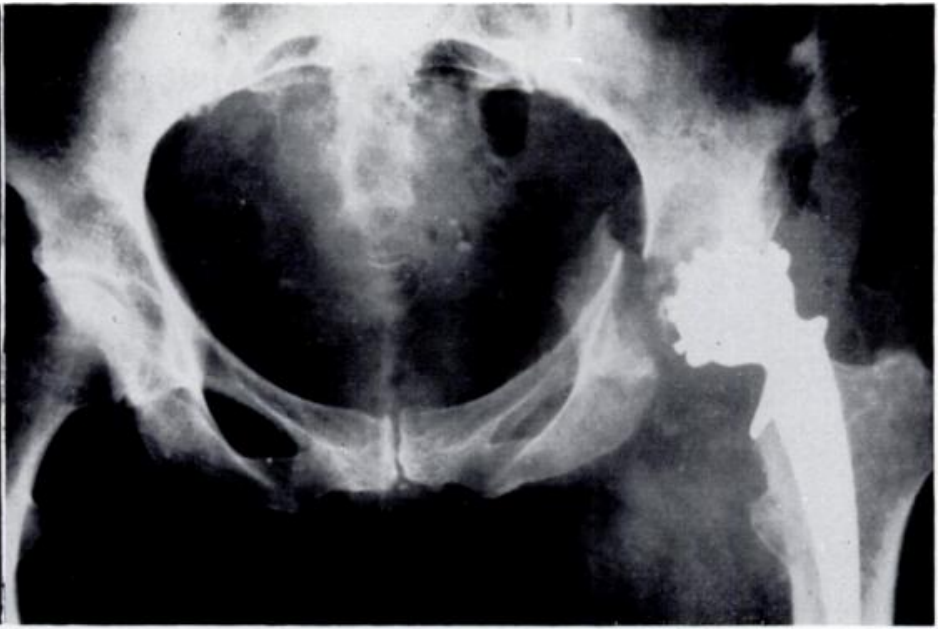

Fig. 7

Case 6. Figure 6-A venogram showing narrowing at the junction of the iliac and femoral veins caused by a deep vein thrombosis. Figure $7-A$ radiograph taken eleven months after Figure 6 . It shows the fracture in bone which previously appeared normal.

radiographs showed a fracture through the ischio-pubic region of the acetabulum (Fig. 8). She continued using her leg but the pain became more severe and in June 1970 the prosthesis was removed. At operation it was noted that the acetabular floor was in four separate parts and was egg-shell thin. All the bony surfaces were pale and appeared avascular. After operation she progressed satisfactorily and has a painless hip and good movement and gets about well with elbow crutches.

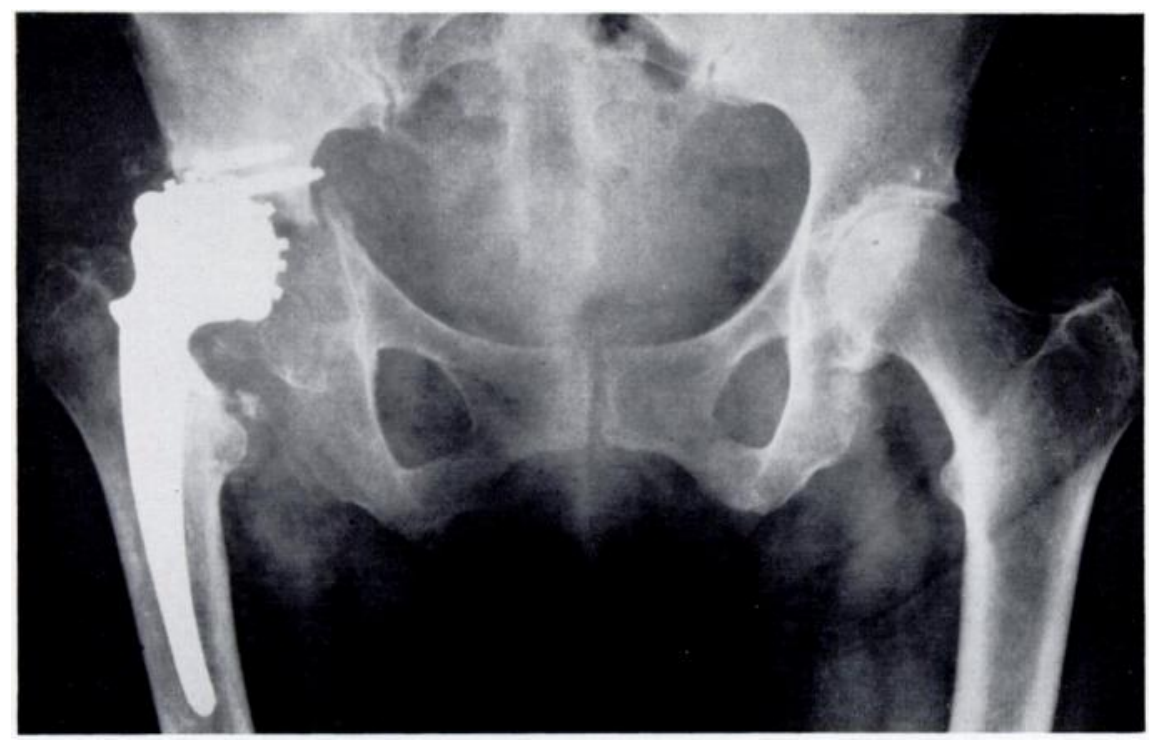

FIG. 8

Case 7-A fracture of the acetabulum eleven months after a McKec arthroplasty.

Case 8-An obese woman of sixty-three had severe osteoarthritis of the right hip. In March 1969 a right McKee arthroplasty was done. She developed a deep vein thrombosis soon after operation, but apart from this progressed reasonably well. In January 1970 she developed pain in the right hip while in bed. Radiographs showed a subluxed cup with a fracture through the pelvic floor (Fig. 9). The prosthesis was removed and the patient was left with a pseudarthrosis. Her course after operation was uneventful. She now has a stable hip and walks well with two sticks.

VOL. 54 B, NO. 4, NOVEMBER 1972 
604

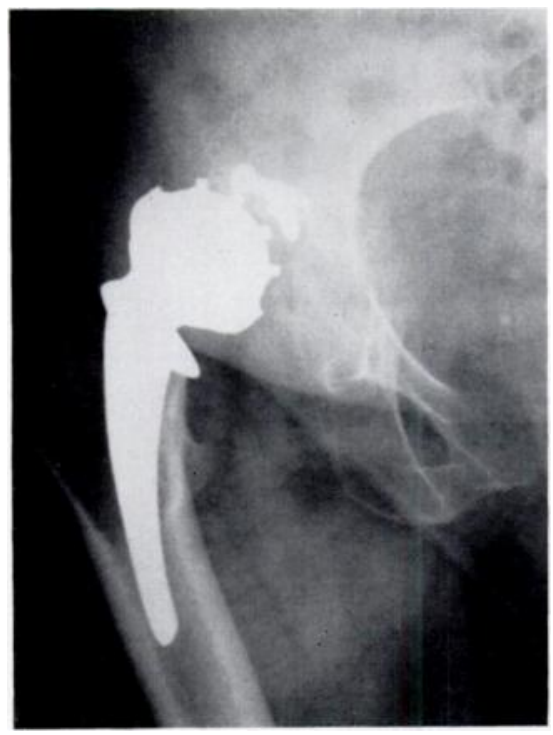

FIG. 9

Case 8- This radiograph shows a subluxed cup and a fracture of the acetabulum. At the operation for removal of the cup the fracture was found to pass across the wall of the acetabulum with independent movement at the fracture site.

\section{A. J. MILLER}

Case 9-A woman of seventy-three with osteoarthritis of her left hip was treated in September 1970 by Ring arthroplasty. Her progress was satisfactory for over a year and in September 1971 she was walking without a stick and had no pain. However, radiographs showed a fracture of the acetabulum (Fig. 10). During the following weeks her hip became painful (Fig. 11) and she was admitted to hospital for investigation and rest. These investigations all proved normal, except for an erythrocyte sedimentation rate of 22 millimetres in the first hour. Although the pain settled down, it recurred when she started to take weight on her leg, and in December 1971 the prosthesis was removed. This case is particularly interesting in that the fracture was noted on routine follow-up when this patient was not complaining of any symptoms.

Comments-Cases 1, 2, 3 and 9 were investigated for metastatic and metabolic bone disease but no abnormality was found. At the second operation on all patients large quantities of dark necrotic tissue was seen surrounding the prosthesis; swabs were taken in Cases 1, 2, 3 and 7 but these proved to be sterile. Histological examination of the capsule from the same cases showed amorphous eosinophilic material with a fibrous appearance and necrotic debris.

The histology of acetabular bone from Case 1 showed degenerate bone with no evidence of secondary neoplasm or foreign material. The bone from Case 7 was normal in one specimen and necrotic in another. Bone from the acetabular floor of Case 8 showed normal trabeculae.

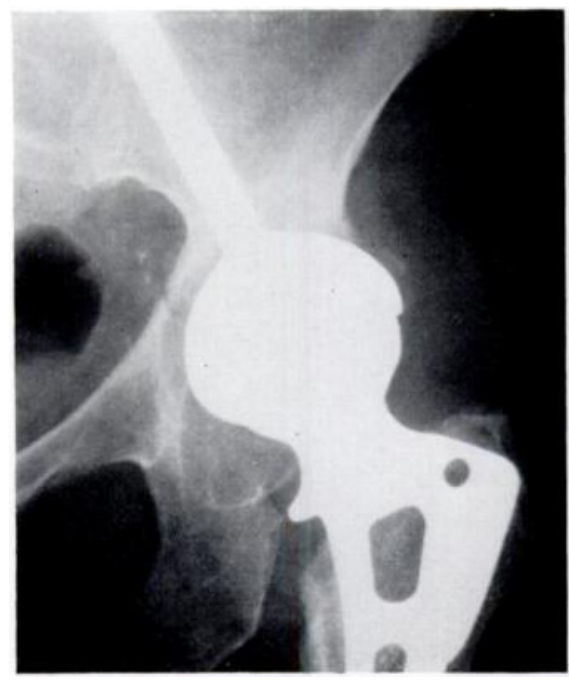

FIG. 10

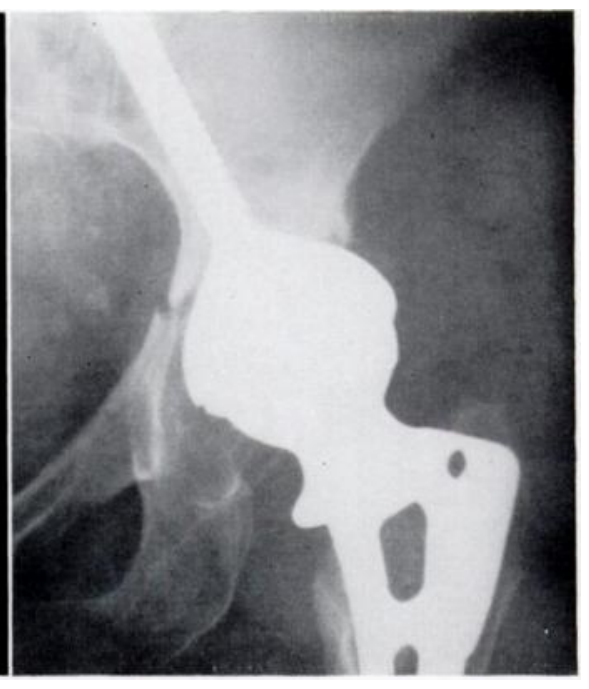

FIG. 11

Case 9-A routine radiograph taken one year after a Ring arthroplasty showed a developing fracture (Fig. 10) and, three weeks later, the fracture has become more obvious (Fig. 11).

\section{DISCUSSION}

The operations were performed by five consultant orthopaedic surgeons working in different centres, all using a standard procedure with which they were familiar. All the patients were women, of whom four were overweight. 
Except for the woman in Case 4 all the patients progressed well for over a year after the operation before unexpectedly presenting with various complications which were infections in Cases 4, 5 and 6, thrombophlebitis or deep vein thrombosis in Cases 1, 2 and 6 and pain from fracture of the ischio-pubic region of the acetabulum in all cases. The patient in Case 6 also had had a fall but there had been no injury in the others. Apart from infection no bone disease was found and it was excluded by biopsy in Cases 1,2, 3,7 and 8. The complications of infection and dislocation are not new (Roles 1971).

Acute infection appeared to contribute to the fracture in Cases 4, 5 and 6. In Case 4 it became evident only four months after the arthroplasty and although the patient responded to antibiotics and drainage at first, the infection recurred and the prosthesis was removed two years later. During this time serial radiographs showed changes gradually occurring in the ischio-pubic region of the acetabulum, leading to eventual fracture. The patient in Case 5 did very well for more than four years before getting the pain, swelling and a fever from infection. Again the prosthesis had to be removed but before this radiographs showed porosis of the acetabulum, and the fracture was seen at operation. It is likely that the infection was blood-borne.

The patient in Case 6 developed a definite infection in the hip one year after a deep vein thrombosis which occurred eight months after operation. This led to a fracture of the acetabulum and removal of the prosthesis. Two other patients developed late deep vein thrombosis on the side of the arthroplasty. The patient in Case 1 was noted to have a swollen leg and foot-drop one year after operation; in Case 2 a swollen, warm leg one year after arthroplasty was thought to be due to thrombophlebitis; the patient also developed a foot-drop a few months later. None of the three patients was treated with antibiotics for the thromboses and the trouble settled down with bed rest, elevation and elastic stockings. However, the episodes left a tendency for swelling to develop in the affected leg. Possibly they all had had a sub-acute infection which caused the deep vein thrombosis.

The patients in Cases 1 and 2 had no further trouble until they developed spontaneous pain in the hip and fracture of the acetabulum. No infection was present and swabs taken at operation were sterile. No antibiotics had been given before operation. It is unlikely that infection was the cause of fracture in these two cases.

Stress fracture-Stress fracture of the normal pelvis has been reported (Devas 1961). The insertion of a total hip prosthesis may alter the mechanical stresses that occur and which are not fully understood. All fractures were in a similar position in the ischio-pubic region and perhaps operation increases the liability of this part to sustain a stress fracture. The patients were all women; the female pelvis is broader and shallower than the male pelvis, and the acetabulum is situated farther from the midline. This may be a factor because of the relatively greater force acting through the acetabulum on weight-bearing; also this force will be increased if the patient is overweight, as were Cases 2, 3, 5 and 8 .

Other factors may be over-reaming of the acetabulum in the Ring arthroplasty or the drilling of the acetabulum for the McKee arthroplasty. The cement used in the insertion of the McKee prosthesis sets at 80 degrees Celsius, which may cause necrosis of the adjacent bone. In the Ring arthroplasty cement was not used but the friction from the drill reaming out the acetabulum has been seen to cause great heat which also might cause necrosis of the underlying bone.

The vascular supply to the pelvis comes from the medullary vessels or the periosteum. In the region of the fracture the periosteal blood supply is from vessels on the inner side of the pelvis where the obturator internus muscle arises. It may be that some of the factors already mentioned-heat of cement, heat of reamer, or over-drilling of the acetabulum-will interfere with the medullary blood supply, and that the periosteal supply is not adequate. Necrosis of bone may then occur and, during the period of revascularisation, the liability to fracture is increased.

VOL. 54 B, NO. 4, NOVEMBER 1972 
The elasticity of bone is greater than that of cement or of the acetabular component of the Ring prosthesis. Recently arthrography has been used to study pain following total hip replacement (Salvati, Freiberger and Wilson 1971). The integrity of the junction of the acrylic cement with the bone interface can be shown by injecting contrast media into the artificial hip. In five hips out of a total of $220 \mathrm{McKee}$ replacements loosening of the cup was demonstrated. This loosening and movement may occur because of the difference in elasticity between the bone and cement, and the persistent movement could erode the surrounding bone, further weakening the pelvis.

The acetabular component of the Ring prosthesis also has a different elasticity from that of bone. If it lies in the weight-bearing line of the pelvis the movement that occurs will tend to push the cup proximally. However, if the stem is not inserted in the weight-bearing line stresses may be set up. These stresses could lead to fracture.

No fracture at the acetabulum level has been reported with any prosthesis having a plastic cup such as the Charnley prosthesis (Charnley 1972). It is possible that the shockabsorbing qualities of the plastic cup may help to avoid this complication.

Removal of the prosthesis leaves the patient with a Girdlestone excision arthroplasty; the results of this as a whole are satisfactory (Taylor 1950).

\section{SUMMARY}

1. Nine cases of stress fracture of the pelvis after total hip replacement are reported, five after Ring replacement and four after a McKee. In none of the cases did the fractures unite.

2. In three cases infection was probably an important cause of the fracture. In six cases there was no infection. Only one presented a history of injury.

3. The combination of the various factors that might have caused the stress fractures is discussed.

I am indebted to $\mathrm{Mr} \mathrm{J}$. G. Bonnin for much helpful advice in the preparation of this paper, and also to Professor James Ellis, Mr G. E. Dunkerley, Mr J. A. Wilkinson, Mr M. S. Brett and Mr P. A. Ring for their kind assistance.

\section{REFERENCES}

Charnley, J. (1972): Personal communication.

Devas, M. B. (1961): Compression Stress Fractures in Man and the Greyhound. Journal of Bone and Joint Surgery, 43-B, 540.

Roles, N. C. (1971): Infection in Total Prosthetic Replacement of the Hip and Knee Joints. Proceedings of the Royal Society of Medicine, 64, 636.

Salvati, E. A., Freiberger, R. H., and Wilson, P. D., Jun. (1971): Arthrography for Complications of Total Hip Replacement. Journal of Bone and Joint Surgery, 53-A, 701.

TAYLOR, R. G. (1950): Pseudarthrosis of the Hip Joint. Journal of Bone and Joint Surgery, 32-B, 161. 